\title{
Stability and synchronization of delayed fractional-order projection neural network with piecewise constant argument of mixed type
}

\author{
Swati Tyagi and Syed Abbas* \\ School of Basic Sciences, Indian Institute of Technology, Mandi, H.P., 175001, India. \\ E-mail: sabbas.iitk@gmail.com
}

\begin{abstract}
Projection equations arise in several optimization problems and possess significant applications in many areas of science and engineering. In this paper, we propose a fractional-order projection neural network to solve quadratic programming problems. We study stability and synchronization for a class of delayed projection neural networks of mixed type via impulsive control. Using concepts of fractional calculus, we investigate the existence of solution and study its global asymptotic stability. Moreover, we propose an effective impulsive control scheme to achieve synchronization for the system. We demonstrate the validity and transient behaviour of the proposed neural network with the help of suitable examples.
\end{abstract}

2010 Mathematics Subject Classification. 26A33. 34D23, 90C20, 34D06

Keywords. Fractional-order; Projection Neural Network; Mittag-Leffler stability; Piecewise constant argument of generalized type; Synchronization.

\section{Introduction}

In recent years, many algorithms have been developed to solve constrained quadratic optimization due to its tremendous application in various scientific and engineering areas such as signal processing, image processing, pattern recognition and optimal control. Some of these algorithms include active set methods [1], simplex method for linear programming [2] and interior point methods for non linear optimization [3]. However, in real world applications, due to time varying characteristics of the optimization problems, it becomes difficult to obtain the optimal solution efficiently using conventional algorithms due to high computation time. Thus artificial neural networks have emerged as one of the most promising approach as, due to inherent massive parallelism it can solve optimization problems in run time much lesser than compared to the traditional algorithms [4]. In [5], neural network has been introduced for the first time to solve closed-loop circuit optimization problems, which promoted the use of neural networks for solving linear and nonlinear programming problems. In [6], Kennedy and Chua studied a neural network to solve nonlinear programming problems by using finite penalty parameter method and discussed the convergence of network to an approximate optimal solution. Thereafter several neural networks have been studied extensively to solve various quadratic programming problems. For more details, we refer to [7-11].

To be more specific, the projection approach has received a remarkable attention in recent years in designing of neural networks for constrained optimization since many constrained optimization problems can be converted into equivalent linear projection equations which are easy to analyze.

\footnotetext{
${ }^{*}$ Corresponding author
} 
In [12], Xia and Wang proposed a projection neural network model to solve monotone variational inequalities and related optimization problems. In [13], Hu and Wang developed a projection neural network to solve pseudomonotone variational inequality and pseudoconvex problems. Thereafter, based on projection methods, several projection neural networks have been proposed and developed to solve linear and quadratic programming problems (for instance, see [14-19]). Their approaches mainly deal with inequality constraints, which are converted into equality constraints by adding slack or surplus variable. These neural networks are endowed with well convergence properties, which converge to exact optimal solution globally. However, in signal transmission of hardware implementation, time delays occur inevitably. This presence of delay affects the dynamical behaviour of the system and may lead to oscillation phenomenon and instability of the network [20,21]. As a result, several projection neural networks with delay have been proposed to solve optimization problems.

In addition to this, projection neural networks have emerged out as a promising tool in the field of fractional calculus also. Due to wide application in almost all branches of sciences, fractional differential equations have received remarkable attention in the field of mechanics, physics, chemistry, informatics, materials and several other applications [22,23]. To the best of our knowledge, very few results are available in literature for projection neural networks in the field of fractional calculus $[24,25]$. This work provides a deep analysis of stability and synchronization for a class of delayed fractional-order projection neural network. Motivated by the above discussion, this paper is devoted to study of quadratic programming problem, which can be described by a fractional-order delayed projection neural network. The novelty and main contribution of this paper is as follows:

- A fractional-order projection neural network with delay and piecewise constant argument of generalized type has been proposed.

- Since the delay term has been incorporated into the model, thus it becomes more realistic to real world applications or quadratic programming problem.

- The proposed neural network model has been proved to be globally Mittag-Leffler stable and globally asymptotically synchronized. Thus the network converges at a rate much faster than the methods derived till now.

The structure of this paper is as follows. Section 2 is divided into two subsections. Subsection 2.1 gives the description of the system model and subsection 2.1 gives some necessary preliminaries. Section 3 gives the proof of the existence of the equilibrium point of the proposed neural network. Section 4 addresses the global synchronization of the proposed model. Section 5 provides some suitable examples to demonstrate the validity of the obtained theoretical results.

\section{Problem formulation}

\subsection{Projection Neural network}

In this section, we provide some necessary mathematical background, which is employed to propose the desired neural network model and further investigate its stability. The projection dynamical system proposed by Friesz et al. in [26] can be described by the following differential equation;

$$
\frac{d x}{d t}=\lambda\left\{P_{\Omega}(x-\mu F(x))-x\right\}
$$


where $\mu$ and $\lambda$ are constants, $\Omega$ is bounded non-empty closed convex subset of $R^{n}, F(x)$ is continuously differentiable vector-valued function from $R^{n}$ to $R^{n}$ and $P_{\Omega}: R^{n} \rightarrow \Omega$ is projection operator defined by

$$
P_{\Omega}(x)=\operatorname{argmin}_{y \in \Omega}\|x-y\| .
$$

Let $\Omega=\left\{x \in R^{n} \mid d_{i} \leq x_{i} \leq h_{i}, i=1,2, \cdots, n\right\}$, then $P_{\Omega}=\left[P_{\Omega}\left(x_{1}\right), \cdots, P_{\Omega}\left(x_{n}\right)\right]^{T}$ is described by the following piecewise linear function:

$$
P_{\Omega}\left(x_{i}\right)=\left\{\begin{array}{ll}
d_{i} & x_{i}<d_{i} \\
u_{i} & d_{i} \leq x_{i} \leq h_{i} . \\
h_{i} & x_{i}>h_{i}
\end{array} .\right.
$$

In [14], the following linear projection neural network model for solving linear projection equation $P_{\Omega}(u-\alpha(M u+q))=u$ is discussed;

$$
\frac{d u}{d t}=\lambda\left\{-u+P_{\Omega}(u-\alpha(M u+q))\right\},
$$

where $\lambda$ is a scaling constant. Under the assumption of matrix $M$ to be symmetric and positivesemidefinite, system (2.3) has been proved to be globally convergent to the equilibrium point and globally asymptotically and exponentially convergent. It has been shown in $[8,27]$ that the projected dynamical systems are significantly important for solving linear and nonlinear monotone variational inequalities and relaxed convex optimization problems. Moreover, many researchers have used discretization scheme to study various neural networks (for instance, see [28-30]). Therefore, it becomes important to develop new neural networks, possessing the above good performance characteristics, to solve a broader class of optimization problems.

Consider the following quadratic programming problem:

$$
\begin{aligned}
& \min \frac{1}{2} x^{T} Q x+c^{T} x \\
& \text { subject to } g \leq B x \leq h,
\end{aligned}
$$

In [27], using the projection formulation [31], the above inequality (2.4) has been converted into following equation:

$$
D x=P_{X}\left(D x-D^{-1} Q x-D^{-1} C\right) .
$$

Under the assumption of matrix $Q \leq Q \leq \bar{Q}$, an interval projection neural network has been presented to solve interval quadratic programming and analyze its stability.

In this paper, we propose the following delayed fractional-order projection neural network with piecewise constant argument of generalized type:

$$
\begin{aligned}
{ }^{C} D_{t}^{\alpha} x(t) & =P_{X}\left(B x(t-\tau)-B^{-1} Q x(\gamma(t))-B^{-1} C\right)-B x(t), \quad t>t_{0} \\
x(t) & =\varphi(t), \quad t \in\left[-\tau, t_{0}\right],
\end{aligned}
$$

where $X$ is a non-empty bounded closed convex set of $R^{n}$. We can rewrite system (2.5) as

$$
\begin{aligned}
{ }^{C} D_{t}^{\alpha} x_{i}(t) & =P_{X}\left(b_{i} x_{i}(t-\tau)-\frac{1}{b_{i}} \sum_{j=1}^{n} q_{i j} x_{j}(\gamma(t))-\frac{1}{b_{i}} c_{i}\right)-b_{i} x_{i}(t), \quad t>t_{0} \\
x_{i}(t) & =\varphi_{i}(t), \quad t \in\left[-\tau, t_{0}\right], \quad i, j=1,2, \cdots, n .
\end{aligned}
$$


where ${ }^{C} D_{t}^{\alpha}$ denotes the Liouville-Caputa fractional-order derivative of order $\alpha, 0<\alpha<1, \tau \geq 0$ denotes the transmission delay, $\varphi(t)$ is continuous for $t \in\left[t_{0}-\tau, t_{0}\right] . Q$ is $n \times n$ symmetric matrix, $B=\operatorname{diag}\left(b_{1}, \cdots, b_{n}\right)$ is positive definite diagonal matrix and $\gamma(t)$ is the deviating argument. We set $\gamma(t)=\xi_{k}$ on $\left[\theta_{k}, \theta_{k+1}\right)$. For $\theta_{k} \leq t<\xi_{k}, \gamma(t)>t$, and thus the system (2.6) consists of advanced argument. Similarly, for $\xi_{k}<t<\theta_{k+1}, \gamma(t)<t$ and the system (2.6) is of delayed argument. Thus the considered system (2.6) of mixed type.

Since the deviating argument $\gamma(t)$ is discontinuous, the right hand side of (2.6) possesses discontinuity at the moments $\theta_{k}, k \in N$. Consequently, we consider the solution of the equations as functions, which are continuous and continuously differentiable within the intervals $\left[\theta_{k}, \theta_{k+1}\right)$. Namely, by a solution $x(t)=\left(x_{1}(t), \cdots, x_{n}(t)\right)^{T}$ of $(2.6)$, we mean a continuous function on $R^{+}$, such that the derivatives exists at each point $t \in R^{+}$, with the possible exception of points $\theta_{k}$, where one sided derivatives exists, and $x(t)$ satisfies the differential equation (2.6) on each interval $\left(\theta_{k}, \theta_{k+1}\right)$ as well. The optimization techniques employed are the projection methods [31] and stability analysis is based on direct method of Lyapunov [32].

\subsection{Preliminaries}

Now we briefly discuss some related definitions and lemmas for later discussion.

Definition 2.1. [33] The Riemann-Liouville fractional integral of fractional-order $\alpha>0$ of a function $f(t)$ is defined as

$$
I_{t}^{\alpha} f(t)=\frac{1}{\Gamma(\alpha)} \int_{t_{0}}^{t}(t-s)^{\alpha-1} f(s) d s,
$$

where $\Gamma($.$) is the Gamma function and is given by \Gamma(z)=\int_{0}^{\infty} t^{z-1} e^{-t} d t$.

Definition 2.2. [33] The Liouville-Caputa derivative of fractional order $\alpha$ of a function $f(t)$ is defined as

$$
{ }^{C} D_{t}^{\alpha} f(t)=\frac{1}{\Gamma(n-\alpha)} \int_{t_{0}}^{t}(t-s)^{n-\alpha-1} f^{(n)}(s) d s
$$

where $n-1<\alpha<n \in \mathbb{Z}^{+}$.

Remark 2.3. 1. For $\alpha=n$, the Liouville-Caputa fractional-order derivative of order $\alpha$ for a function $f(t)$ is similar to usual integer-order derivative $f^{(n)}(t)$.

2. The Liouville-Caputa fractional derivative of a constant is zero.

3. The initial time considered in ${ }^{C} D_{t}^{\alpha} f(t)$ is from $t_{0}$.

Proposition 2.4. [27] Let $x^{*}$ be the solution of the projection equation

$$
B x=P_{X}\left(B x-B^{-1} Q x-B^{-1} C\right) .
$$

Then $x^{*}$ is an optimal solution of the quadratic problem (2.4).

Definition 2.5. [27] The point $x^{*}=\left(x_{1}^{*}, x_{2}^{*}, \cdots, x_{n}^{*}\right)^{T}$ is said to be an equilibrium point of the delayed projection neural network (2.5), if it satisfies

$$
0=P_{X}\left(B x^{*}-B^{-1} Q x^{*}-B^{-1} C\right)-B x^{*} .
$$


From Proposition 2.4 and Definition 2.5, we have the following theorem.

Theorem 2.6. The point $x^{*}$ is an equilibrium point of the delayed projection neural network $(2.5)$ if and only if it is an optimal solution of the quadratic problem (2.4).

Lemma 2.7. [31] Let $\Omega \subset R^{n}$ be a closed convex set. Then,

$$
\begin{aligned}
& \left(v-P_{\Omega}(v)\right)^{T}\left(P_{\Omega}(v)-u\right) \geq 0, \quad \forall u \in \Omega, v \in R^{n}, \\
& \left\|P_{\Omega}(u)-P_{\Omega}(v)\right\| \leq\|u-v\|, \quad \forall u, v \in R^{n},
\end{aligned}
$$

where $P_{\Omega}(u)$ represents the projection function on $\Omega$.

Lemma 2.8. [24] If $h(t) \in C^{1}([0,+\infty), R)$ denotes a continuously differentiable function, then the following inequality holds almost everywhere:

$$
D_{t}^{\alpha}|h(t)| \leq \operatorname{sgn}(h(t)) D_{t}^{\alpha} h(t), \quad 0<\alpha \leq 1 .
$$

Lemma 2.9. [34] Let $V(t)$ be a continuous function on $\left[t_{0}, \infty\right)$ satisfying

$$
{ }^{C} D_{t}^{\alpha} V(t) \leq-\gamma V(t), \quad \alpha \in(0,1)
$$

for some constant $\gamma$. Then

$$
V(t) \leq V\left(t_{0}\right) E_{\alpha}\left(-\gamma\left(t-t_{0}\right)^{\alpha}\right), \quad t \geq t_{0}
$$

\section{Existence of solution}

In this section, we prove that there exists a unique solution to the delayed projection neural network (2.6). To prove main results, we assume that the system (2.6) satisfies following assumptions:

A1: $\frac{\left(\sigma_{1}+\sigma_{2}\right)}{N^{\alpha}}<1$, where $\sigma_{1}=2 \bar{b}=2 \max _{i}\left\{\left|b_{i}\right|\right\}$ and $\sigma_{2}=q^{*}=\max _{i}\left\{\frac{1}{\left|b_{i}\right|} \sum_{j=1}^{n}\left|q_{j i}\right|\right\}$.

$\left.\mathrm{A} 2: \bar{l}=\max _{i}\left\{l_{i}\right\}, V_{i}\left(t^{+}, e_{i}(t)+L_{k} e_{i}(t)\right)\right) \leq V_{i}\left(t, e_{i}(t)\right)$.

A3: $\mu_{1}=\min _{1 \leq i \leq n}\left(\left|b_{i}\right|-\rho \frac{1}{\left|b_{i}\right|} \sum_{j=1}^{n}\left|q_{j i}\right|-\left|l_{i}\right|\right)>\mu_{2}=\max _{1 \leq i \leq n}\left(\left|b_{i}\right|\right)>0$, where $\rho$ is given by $(4.5)$.

We define norm as $\|x(t)\|=\sum_{i=1}^{n} \sup _{t}\left\{e^{-N t}\left|x_{i}(t)\right|\right\}$.

Theorem 3.1. If Assumption (A1) holds; then there exists a unique equilibrium point for the system (2.6). 
Proof. Fix $k \in \mathbb{N}$ and consider the case $\theta_{k} \leq \xi_{k} \leq t_{0} \leq \theta_{k+1}$.

First, we prove that for every $\left(t_{0}, x_{0}\right) \in\left[\theta_{k}, \theta_{k+1}\right] \times R^{n}$, there exists a unique solution $x(t)=$ $x\left(t, t_{0}, \varphi\left(t_{0}\right)\right)$. From (2.6), consider the following equivalent integral equation

$$
\begin{aligned}
x_{i}(t)= & \varphi_{i}\left(t_{0}\right)+\frac{1}{\Gamma(\alpha)} \int_{t_{0}}^{t}(t-s)^{\alpha-1}\left\{-b_{i} x_{i}(s)\right. \\
& \left.+P_{X}\left(b_{i} x_{i}(s-\tau)-\frac{1}{b_{i}} \sum_{j=1}^{n} q_{i j} x_{j}\left(\xi_{k}\right)-\frac{1}{b_{i}} c_{i}\right)\right\} d s .
\end{aligned}
$$

Now we construct the sequences $x_{i}^{m}(t), \cdots, x_{i}^{0}(t), i=1,2, \cdots, m$. Thus we have

$$
\begin{aligned}
x_{i}^{m+1}(t)= & \varphi_{i}\left(t_{0}\right)+\frac{1}{\Gamma(\alpha)} \int_{t_{0}}^{t}(t-s)^{\alpha-1}\left\{-b_{i} x_{i}^{m}(s)\right. \\
& \left.+P_{X}\left(b_{i} x_{i}^{m}(s-\tau)-\frac{1}{b_{i}} \sum_{j=1}^{n} q_{i j} x_{j}^{m}\left(\xi_{k}\right)-\frac{1}{b_{i}} c_{i}\right)\right\} d s .
\end{aligned}
$$

From equation (3.2), for any two sequences $x_{i}^{m+1}$ and $x_{i}^{m}$, we have

$$
\begin{aligned}
x_{i}^{m+1}(t)-x_{i}^{m}(t)= & \frac{1}{\Gamma(\alpha)} \int_{t_{0}}^{t}(t-s)^{\alpha-1}\left\{-b_{i}\left(x_{i}^{m}(s)-x_{i}^{m-1}(s)\right\} d s\right. \\
+ & \frac{1}{\Gamma(\alpha)} \int_{t_{0}}^{t}(t-s)^{\alpha-1}\left\{P_{X}\left(b_{i} x_{i}^{m}(s-\tau)-\frac{1}{b_{i}} \sum_{j=1}^{n} q_{i j} x_{j}^{m}\left(\xi_{k}\right)-\frac{1}{b_{i}} c_{i}\right)\right. \\
& \left.-P_{X}\left(b_{i} x_{i}^{m-1}(s-\tau)-\frac{1}{b_{i}} \sum_{j=1}^{n} q_{i j} x_{j}^{m-1}\left(\xi_{k}\right)-\frac{1}{b_{i}} c_{i}\right)\right\} d s .
\end{aligned}
$$

Taking modulus both sides, we obtain

$$
\begin{aligned}
\left|x_{i}^{m+1}(t)-x_{i}^{m}(t)\right| \leq & \frac{1}{\Gamma(\alpha)} \int_{t_{0}}^{t}(t-s)^{\alpha-1}\left\{\left|b_{i}\right|\left|x_{i}^{m}(s)-x_{i}^{m-1}(s)\right|\right\} d s \\
& +\frac{1}{\Gamma(\alpha)} \int_{t_{0}}^{t}(t-s)^{\alpha-1}\left\{\left|b_{i}\right|\left|x_{i}^{m}(s-\tau)-x_{i}^{m-1}(s-\tau)\right|\right\} d s \\
& +\frac{1}{\Gamma(\alpha)} \int_{t_{0}}^{t}(t-s)^{\alpha-1}\left\{\frac{1}{\left|b_{i}\right|} \sum_{j=1}^{n}\left|q_{i j}\right|\left|x_{j}^{m}\left(\xi_{k}\right)-x_{j}^{m-1}\left(\xi_{k}\right)\right|\right\} d s .
\end{aligned}
$$

For some constant $N>0$, multiplying by $e^{-N t}$ both sides of (3.4) and using definition of norm, we 
obtain

$$
\begin{aligned}
\| & x^{m+1}(t)-x^{m}(t) \|=\sum_{i=1}^{n}\left\{e^{-N t}\left|x_{i}^{m+1}(t)-x_{i}^{m}(t)\right|\right\} \\
\leq & \sum_{i=1}^{n} \frac{\left|b_{i}\right|}{\Gamma(\alpha)} \int_{t_{0}}^{t}(t-s)^{\alpha-1} e^{-N(t-s)}\left\{e^{-N s}\left|x_{i}^{m}(s)-x_{i}^{m-1}(s)\right|\right\} d s \\
& +\sum_{i=1}^{n} \frac{\left|b_{i}\right|}{\Gamma(\alpha)} \int_{t_{0}}^{\tau}(t-s)^{\alpha-1} e^{-N(t-s+\tau)}\left\{e^{-N(s-\tau)}\left|x_{i}^{m}(s-\tau)-x_{i}^{m-1}(s-\tau)\right|\right\} d s \\
& +\sum_{i=1}^{n} \frac{\left|b_{i}\right|}{\Gamma(\alpha)} \int_{\tau}^{t}(t-s)^{\alpha-1} e^{-N(t-s+\tau)}\left\{e^{-N(s-\tau)}\left|x_{i}^{m}(s-\tau)-x_{i}^{m-1}(s-\tau)\right|\right\} d s \\
& +\sum_{i=1}^{n} \sum_{j=1}^{n} \frac{1}{\left|b_{i}\right| \Gamma(\alpha)}\left|q_{j i}\right| \int_{t_{0}}^{t}(t-s)^{\alpha-1} e^{-N(t-s)}\left\{e^{-N s} \mid\left(x_{i}^{m}\left(\xi_{k}\right)-x_{i}^{m-1}\left(\xi_{k}\right) \mid\right\} d s\right. \\
\leq & \frac{\bar{b}}{N^{\alpha}} \sum_{i=1}^{n} \sup _{t}\left\{e^{-N t}\left|\varphi_{i}^{m}\left(t_{0}\right)-\varphi_{i}^{m-1}\left(t_{0}\right)\right|+2 \frac{\bar{b}}{N^{\alpha}} \sum_{i=1}^{n} \sup _{t}\left\{e^{-N t}\left|x_{i}^{m}(t)-x_{i}^{m-1}(t)\right|\right.\right. \\
& +\frac{q^{*}}{N^{\alpha}} \sum_{i=1}^{n} \sup _{t}\left\{e^{-N t}\left|x_{i}^{m}(t)-x_{i}^{m-1}(t)\right|\right. \\
\leq & \frac{\left(\sigma_{1}+\sigma_{2}\right)}{N^{\alpha}}\left\|x_{i}^{m}(t)-x_{i}^{m-1}(t)\right\| .
\end{aligned}
$$

Repeating above argument, we obtain

$$
\left\|x^{m+1}(t)-x^{m}(t)\right\| \leq\left(\frac{\left(\sigma_{1}+\sigma_{2}\right)}{N^{\alpha}}\right)^{m}\left\|x^{1}(t)-x^{0}(t)\right\| .
$$

Thus there exists a unique solution $x\left(t, t_{0}, \varphi\left(t_{0}\right)\right)$ on closed interval $\left[\xi_{k}, t_{0}\right]$. Using property of projection map, $x(t)$ can be continued to $\theta_{k+1}$. Since it is a solution to the differential equation (2.6) on $\left[\theta_{k}, \theta_{k+1}\right)$. Using similar argument, we can continue $x(t)$ on any interval $\left(\theta_{k+1}, \theta_{k+2}\right]$. Continuing in similar manner, we obtain that there exists a unique solution to (2.6). This completes the proof.

Q.E.D.

\section{Synchronization}

In this section, we investigate how drive-response fractional-order projection neural network achieve synchronization under linear control. Based on the concept of drive-response system, we refer to the system (2.6) as master (drive) system and characterize the corresponding slave (response) system as follows:

$$
{ }^{C} D_{t}^{\alpha} x_{i}(t)=P_{X}\left(b_{i} x_{i}(t-\tau)-\frac{1}{b_{i}} \sum_{j=1}^{n} q_{i j} x_{j}(\gamma(t))-\frac{1}{b_{i}} c_{i}\right)-b_{i} x_{i}(t)+u_{i}(t),
$$

where $u_{i}(t)$ is impulsive control input. We shall use a linear feedback scheme to realize synchronization between system (2.6) and (4.1), that is, we define controller $u_{i}(t)$ as $u_{i}(t)=L_{i k}\left[x_{i}(t)-\right.$ 
$\left.\eta_{i}(t)\right] \delta\left(t-t_{k}\right)$, where $L_{i k} \in R^{n}$ represents the control gain matrix, and is a diagonal matrix. The discrete set $\left\{t_{k}\right\}$ satisfies $0 \leq t_{0} \leq t_{1} \leq \cdots \leq t_{k} \rightarrow \infty$ as $k \rightarrow \infty$, and $\delta(\cdot)$ is the dirac-delta impulsive function. The error relative to $i^{\text {th }}$ node is given by

$$
{ }^{C} D_{t}^{\alpha} e_{i}(t)=-b_{i} e_{i}(t)+P_{X}\left(e_{i}(\gamma(t))\right)+L_{i k} e_{i}(t),
$$

where

$$
\begin{aligned}
P_{X}\left(e_{i}(\gamma(t))\right)= & P_{X}\left(b_{i} x_{i}(t-\tau)-\frac{1}{b_{i}} \sum_{j=1}^{n} q_{i j} x_{j}(\gamma(t))-\frac{1}{b_{i}} c_{i}\right) \\
& -P_{X}\left(b_{i} \eta_{i}(t-\tau)-\frac{1}{b_{i}} \sum_{j=1}^{n} q_{i j} \eta_{j}(\gamma(t))-\frac{1}{b_{i}} c_{i}\right)
\end{aligned}
$$

Writing (4.2) in compact matrix form, we obtain

$$
\begin{aligned}
{ }^{C} D_{t}^{\alpha} e(t) & =-B e(t)+P_{X}(e(\gamma(t)))+U(t), \quad t \neq t_{k}, t>t_{0}, \\
\Delta e\left(t_{k}\right) & =e\left(t_{k}^{+}\right)-e\left(t_{k}^{-}\right)=L_{k} e\left(t_{k}^{-}\right) .
\end{aligned}
$$

Definition 4.1. [34] The master system (2.6) and slave system (4.1) are said to be globally MittagLeffler synchronized, if for $\varphi \in\left(C\left[t_{0}-\tau, t_{0}\right], R^{n}\right)$, there exists a constant $\lambda>0$ and $\beta>0$, such that $\left\|x\left(t ; t_{0}, \varphi\left(t_{0}\right)\right)-\eta\left(t ; t_{0}, \psi\left(t_{0}\right)\right)\right\| \leq\left\{m\left(\varphi\left(t_{0}\right)-\psi\left(t_{0}\right)\right) E_{\alpha}\left(-\lambda\left(t-t_{0}\right)^{\alpha}\right)\right\}^{\beta}, t \geq t_{0}$, where $m(0)=0$, $m(\varphi) \geq 0$ and $m(\varphi)$ is Lipschitzian with respect to $\varphi$.

Remark 4.2. Global Mittag-Leffler stability implies global asymptotic stability.

Lemma 4.3. Assume that $e(t)$ is the solution of system (4.4). Then under Assumption (A1), for all $t \in R^{+},\|e(\gamma(t))\| \leq \rho\|e(t)\|$, where $\rho$ is given by

$$
\rho=\frac{N^{\alpha}+2 \bar{b}+\bar{l}}{N^{\alpha} \bar{b}-q^{*}}
$$

provided $N^{\alpha} \bar{b} \neq q^{*}$.

Proof. We have

$$
{ }^{C} D_{t}^{\alpha} e_{i}(t)=-b_{i} e(t)+P_{X}\left(e_{i}(\gamma(t))\right)+U_{i}(t) .
$$

Then for $t \in\left[\theta_{k}, \theta_{k+1}\right)$, we have

$$
e_{i}(t)=e_{i}\left(\xi_{k}\right)+\frac{1}{\Gamma(\alpha)} \int_{\xi_{k}}^{t}(t-s)^{\alpha-1}\left[-b_{i} e_{i}(s)+P_{X}\left(e_{i}(\gamma(s))\right)+U_{i}(s)\right] d s,
$$

where $\gamma(t)=\xi_{k}$ if $t \in\left[\theta_{k}, \theta_{k+1}\right)$. Also on $t \in\left[\theta_{k}, \theta_{k+1}\right)$, we have

$$
e_{i}\left(\xi_{k}\right)=e_{i}(t)+\frac{1}{\Gamma(\alpha)} \int_{\xi_{k}}^{t}(t-s)^{\alpha-1}\left[-b_{i} e_{i}(s)+P_{X}\left(e_{i}(\gamma(s))\right)+U_{i}(s)\right] d s .
$$


Taking modulus and multiplying by $\exp (-N t)$ both sides, we obtain

$$
\begin{aligned}
& \sum_{i=1}^{n} \exp (-N t)\left|e_{i}\left(\xi_{k}\right)\right| \\
& \leq \sum_{i=1}^{n} \exp (-N t)\left|e_{i}(t)\right|+\frac{1}{\Gamma(\alpha)} \int_{\xi_{k}}^{t}(t-s)^{\alpha-1} \exp (-N t) \sum_{i=1}^{n}\left|b_{i}\right|\left|e_{i}(s)\right| d s \\
& \quad+\frac{1}{\Gamma(\alpha)} \int_{\xi_{k}}^{t}(t-s)^{\alpha-1} \exp (-N t) \sum_{i=1}^{n}\left|b_{i}\right|\left|e_{i}(s-\tau)\right| d s \\
& \quad+\sum_{i=1}^{n}\left(\frac{\sum_{j=1}^{n}\left|q_{j i}\right|}{\left|b_{i}\right| \Gamma(\alpha)} \int_{\xi_{k}}^{t}(t-s)^{\alpha-1} \exp (-N t)\left|e_{i}\left(\xi_{k}\right)\right| d s\right) \\
& \quad+\frac{1}{\Gamma(\alpha)} \int_{\xi_{k}}^{t}(t-s)^{\alpha-1} \exp (-N t) \sum_{i=1}^{n}\left|l_{i}\right|\left|e_{i}(s)\right| d s .
\end{aligned}
$$

Following similar steps as in Theorem 3.1 and further taking supremum both sides, we obtain

$$
\begin{aligned}
& \left\|e\left(\xi_{k}\right)\right\| \\
& \leq\|e(t)\|+\left(\frac{2 \bar{b}+\bar{l}}{\Gamma(\alpha)}\right) \int_{t_{0}}^{t}(t-s)^{\alpha-1} \exp (-N(t-s))\|e(t)\| d s+\frac{q^{*}}{N^{\alpha}}\left\|e\left(\xi_{k}\right)\right\| \\
& \leq\left(1+\frac{2 \bar{b}+\bar{l}}{N^{\alpha}}\right)\|e(t)\|+\frac{q^{*}}{N^{\alpha}}\left\|e\left(\xi_{k}\right)\right\| .
\end{aligned}
$$

Thus we have

$$
\left\|e\left(\xi_{k}\right)\right\| \leq \rho\|e(t)\|
$$

where $\rho=\frac{1+\frac{2 \bar{b}+\bar{l}}{N^{\alpha}}}{1-\frac{q^{*}}{N^{\alpha}}}>0$. This completes the proof.

Q.E.D.

Now we study the dynamic behaviour of the error $e(t)$ to check for the global asymptotic synchronization of system (4.1).

Theorem 4.4. If Assumption (A2)-(A3) hold and the system parameters $b_{i}, l_{i}, q_{i j}$ satisfy $\mu_{1}, \mu_{2}>$ $0,(i, j=1,2, \cdots, n)$, then the error system (4.4) is globally Mittag-Leffler stable and hence the master system (2.6) and the slave system (4.4) are globally asymptotically synchronized.

Proof. We define Lyapunov function as

$$
V(t)=\sum_{i=1}^{n} V_{i}(t)=\sum_{i=1}^{n}\left|e_{i}(t)\right|
$$


For $t=t_{k}$, we have

$$
\begin{aligned}
V\left(t^{+}, e\left(t^{+}\right)\right) & =\sum_{i=1}^{n} V_{i}\left(t^{+}, e_{i}\left(t^{+}\right)\right) \\
& =\sum_{i=1}^{n} V_{i}\left(t^{+}, e_{i}(t)+L_{k} e_{i}(t)\right) .
\end{aligned}
$$

Thus from Assumption (A2), we obtain

$$
V\left(t^{+}, e\left(t^{+}\right)\right) \leq \sum_{i=1}^{n} V_{i}\left(t, e_{i}(t)\right)=V(t, e(t)) .
$$

Let $t \geq t_{0}$ and $t \in\left[t_{k-1}, t_{k}\right)$, from Lemma 2.8, calculating the fractional-order derivatives of $V(t)$ along the solutions of (4.1), we have

$$
\begin{aligned}
& { }^{C} D_{t^{+}}^{\alpha} V(t, e(t))=\sum_{i=1}^{n} \operatorname{sgn}\left(e_{i}(t)\right)^{C} D_{t}^{\alpha} e_{i}(t), \\
& \leq \sum_{i=1}^{n}\left[-\left|b_{i}\right|\left|e_{i}(t)\right|+\left|b_{i}\right|\left|e_{i}(t-\tau)\right|+\frac{1}{\left|b_{i}\right|} \sum_{j=1}^{n}\left|q_{i j}\right|\left|e_{j}(\gamma(t))\right|+\left|l_{i}\right|\left|e_{i}(t)\right|\right] \\
& \leq \sum_{i=1}^{n}-\left(\left|b_{i}\right|-\rho \frac{1}{\left|b_{i}\right|} \sum_{j=1}^{n}\left|q_{j i}\right|-\left|l_{i}\right|\right)\left|e_{i}(t)\right|+\sum_{i=1}^{n}\left|b_{i}\right|\left|e_{i}(t-\tau)\right| \\
& \leq-\min _{1 \leq i \leq n}\left(\left|b_{i}\right|-\rho \frac{1}{\left|b_{i}\right|} \sum_{j=1}^{n}\left|q_{j i}\right|-\left|l_{i}\right|\right) \sum_{i=1}^{n}\left|e_{i}(t)\right|+\max _{1 \leq i \leq n}\left|b_{i}\right| \sum_{i=1}^{n}\left|e_{i}(t-\tau)\right| \\
& \leq-\mu_{1} V(t, e(t))+\mu_{2} \sup _{t-\tau \leq s \leq t} V(s, e(s)),
\end{aligned}
$$

where $\mu_{1}=\min _{1 \leq i \leq n}\left(\left|b_{i}\right|-\rho \frac{1}{\left|b_{i}\right|} \sum_{j=1}^{n}\left|q_{j i}\right|-\left|l_{i}\right|\right)>0$, and $\mu_{2}=\max _{1 \leq i \leq n}\left|b_{i}\right|>0$.

From the above approximation, for any solution $e(t)$ of (4.2), which satisfies the Razumikhin condition [35],

$$
V(s, e(s)) \leq V(t, e(t)), \quad t-\tau \leq s \leq t
$$

we obtain

$$
{ }^{C} D_{t^{+}}^{\alpha} V(t, e(t)) \leq-\left(\mu_{1}-\mu_{2}\right) V(t, e(t))
$$

From Assumption (A3), for some $\lambda=\mu_{1}-\mu_{2}>0$, it follows that

$$
{ }^{C} D_{t^{+}}^{\alpha} V(t, e(t)) \leq-\lambda V(t, e(t)), \quad t \neq t_{k}, \quad t>t_{0} .
$$


Using Lemma 2.9, we obtain

$$
V(t, e(t)) \leq V\left(t_{0}^{+}, e\left(t_{0}^{+}\right)\right) E_{\alpha}\left(-\lambda\left(t-t_{0}\right)^{\alpha}\right), \quad t \in\left[t_{0}, \infty\right) .
$$

Thus,

$$
\begin{aligned}
\|x(t)-\eta(t)\|=\sum_{i=1}^{n}\left|x_{i}(t)-\eta_{i}(t)\right| & \leq E_{\alpha}\left(-\lambda\left(t-t_{0}\right)^{\alpha}\right) \sum_{i=1}^{n}\left|x_{i}\left(t_{0}^{+}\right)-\eta_{i}\left(t_{0}^{+}\right)\right| \\
& \leq m E_{\alpha}\left(-\lambda\left(t-t_{0}\right)^{\alpha}\right), \quad t>t_{0},
\end{aligned}
$$

where $m=\left\|\varphi\left(t_{0}\right)-\psi\left(t_{0}\right)\right\| \geq 0$ and $m=0$ holds if $x_{i}\left(t_{0}^{+}\right)=\eta_{i}\left(t_{0}^{+}\right)$for $t \in\left[t_{0}-\tau, t_{0}\right]$. From (4.14) and (4.17), we conclude that the trivial solution of (4.4) is globally Mittag-Leffler stable, that is the delayed projection neural network (4.1) is globally exponentially (or globally asymptotically) synchronized.

Q.E.D.

\section{Numerical Examples}

Example 5.1. Consider the following three dimensional fractional-order projective neural network with delay;

$$
\left(\begin{array}{l}
{ }^{C} D_{t}^{\alpha} x_{1}(t) \\
{ }^{C} D_{t}^{\alpha} x_{2}(t) \\
{ }^{C} D_{t}^{\alpha} x_{3}(t)
\end{array}\right)=P_{X}\left\{\left(\begin{array}{l}
x_{1}(t-\tau) \\
x_{2}(t-\tau) \\
x_{3}(t-\tau)
\end{array}\right)-\left(\begin{array}{ccc}
0.84 & 0.008 & 0.24 \\
0.008 & 0.804 & 0.16 \\
0.24 & 0.16 & 0.76
\end{array}\right)\left(\begin{array}{l}
x_{1}(t) \\
x_{2}(t) \\
x_{3}(t)
\end{array}\right)-\left(\begin{array}{c}
1 \\
-1 \\
0.5
\end{array}\right)\right\}-\left(\begin{array}{l}
x_{1}(t) \\
x_{2}(t) \\
x_{3}(t)
\end{array}\right)
$$

where $X=\left\{x_{i} \mid-12 \leq x_{i} \leq 12, i=1,2,3\right\}$ and $\alpha \in(0,1)$. In the absence of any delay, the solution of the projection system (5.1) is asymptotically stable as shown in sub-figure 1(a). But as delay is introduced, solution starts losing its stability. As shown in sub figures 1(b)-1(e), the fractional-order delayed projective system admits a chaotic behavior for $\tau=0.05$ and $\alpha=0.95$. Sub-figure 1(f) shows the time portrait for unstable solution in the presence of delay. 


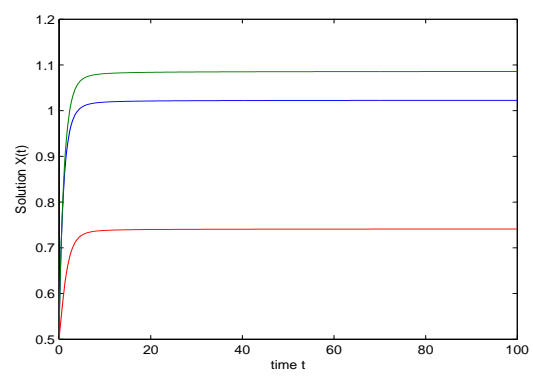

(a) Stable solution in the absence of delay

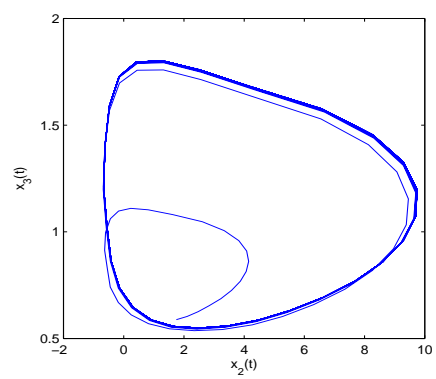

(c) Phase portrait for $\tau=0.05$

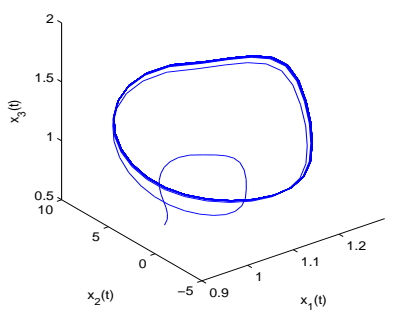

(e) Phase portrait for $\tau=0.05$

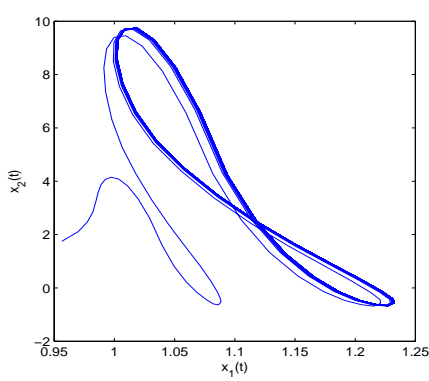

(b) Phase portrait for $\tau=0.05$

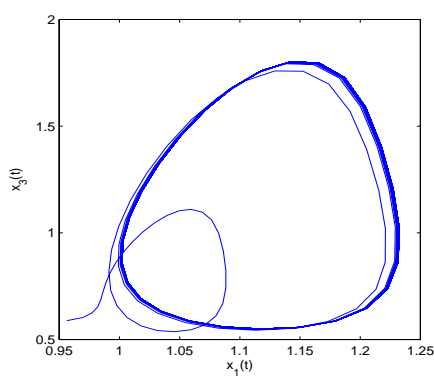

(d) Phase portrait for $\tau=0.05$

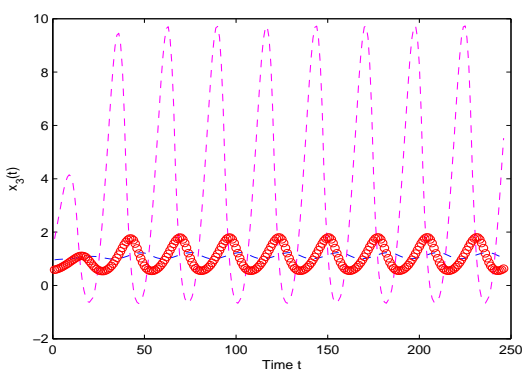

(f) Time portrait showing unstable solution for $\tau=0.05$

Figure 1. Effect of delay on behavior of solutions of delayed fractional-order projection neural network (5.1) for $\alpha=0.95$.

Now, we design the controlled response fractional-order projective neural network by choosing the controller gain matrix $U(t)$ as $L=\operatorname{diag}(10,-5,8)$. It can be easily verified that the synchronization between the original system and the controlled system is achieved. We select initial states as $(4.5,4.5,-2),(-7,2.5,0.5),(0.5,3.5,-3),(-5,-1.5,-8.5),(-1.5,2,-2.5)$. Figure 2 represents the global asymptotic stability of the controlled system to the equilibrium point 
$(11.2228,-3.7996,8.5367)$.
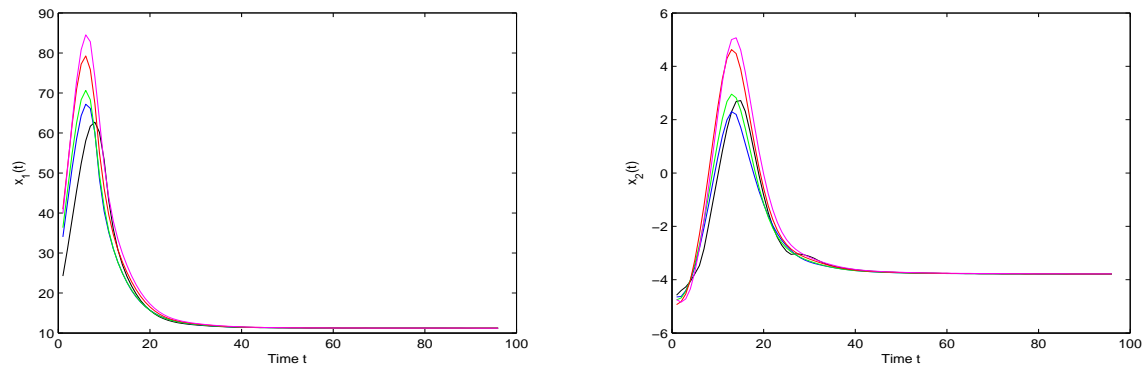

(a) Global asymptotically stable solution $\left(x_{1}(t)\right)$ (b) Global asymptotically stable solution $\left(x_{2}(t)\right)$ in the presence of control in the presence of control

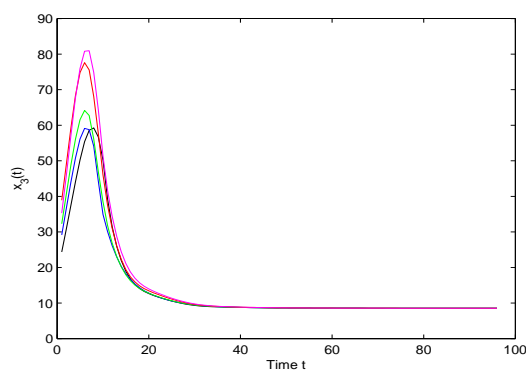

(c) Global asymptotically stable solution $\left(x_{3}(t)\right)$ in the presence of control

FiguRE 2. Global asymptotically stable solutions of system (5.1) in presence of control for $\tau=0.05$ and $\alpha=0.95$.

Example 5.2. Consider the Example (5.1) for the case when matrix $Q$ is not symmetric and other parameters remain unchanged [25].

$$
Q=\left(\begin{array}{ccc}
1.04 & -0.42 & 0.5 \\
-0.10 & 0.92 & -0.06 \\
0.14 & 0.06 & 0.096
\end{array}\right)
$$

In the absence of any delay, the solution of the projection system (5.2) is asymptotically stable (see [25]), but as delay is introduced, solution starts losing its stability. Figure 3 displays the chaotic behavior of the fractional-order delayed projective system for $\tau=0.05$ and $\alpha=0.95$. 


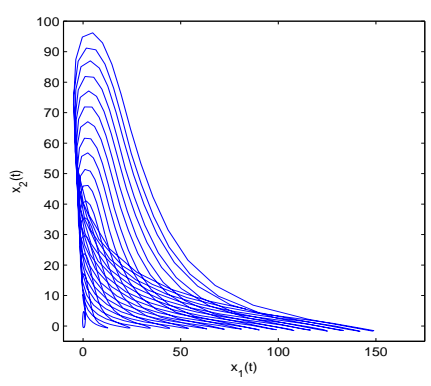

(a) Phase portrait for $\tau=0.05$

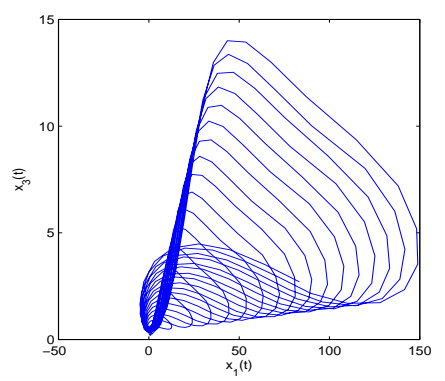

(c) Phase portrait for $\tau=0.05$

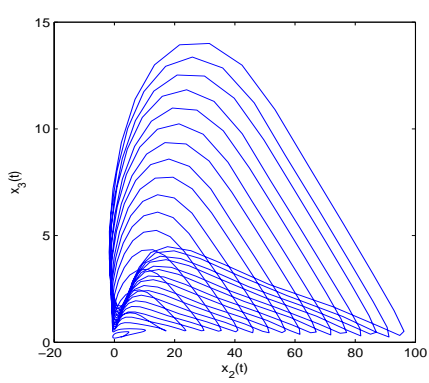

(b) Phase portrait for $\tau=0.05$

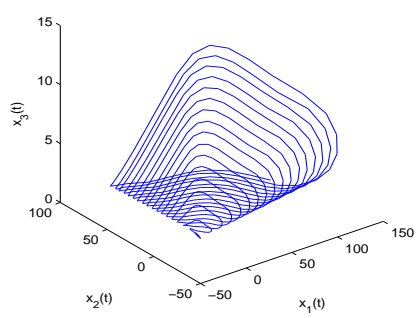

(d) Phase portrait for $\tau=0.05$

Figure 3. Chaotic behaviors of delayed fractional-order projection neural network (5.2) for $\alpha=$ 0.95 .

In this case we choose the controller gain matrix $U(t)$ as $L=\operatorname{diag}(6,-5,-8)$. It can be easily verified that the synchronization between the original system and the controlled system is achieved. Figure 4 represents the global asymptotic stability of the controlled system to the equilibrium point $(-5.44656,-4.80175,-4.49087)$. 

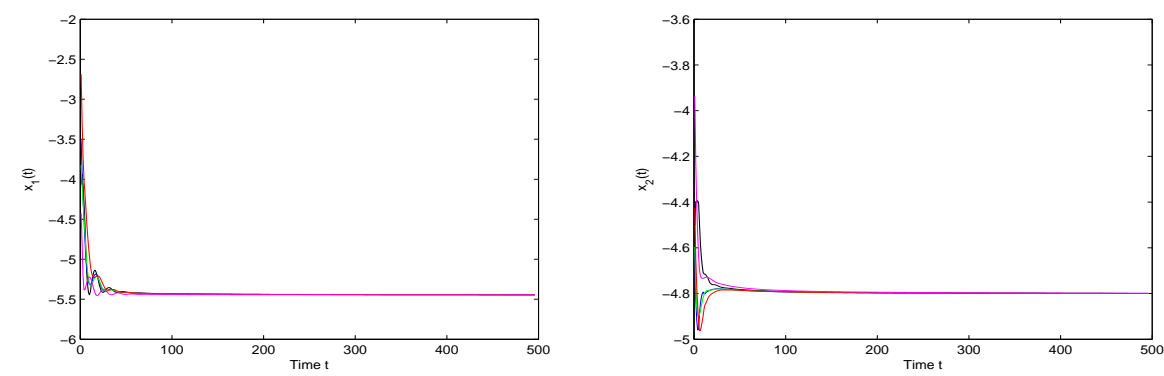

(a) Global asymptotically stable solution $\left(x_{1}(t)\right)$ (b) Global asymptotically stable solution $\left(x_{2}(t)\right)$ in the presence of control in the presence of control

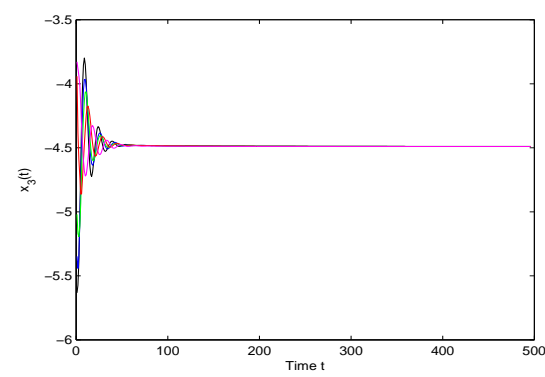

(c) Global asymptotically stable solution $\left(x_{3}(t)\right)$

in the presence of control

FIGURE 4. Global asymptotically stable solutions of system (5.2) in presence of control for $\tau=0.05$ and $\alpha=0.95$.

\section{Discussion}

In this work, we have investigated the qualitative behavior of a fractional order projection neural network with time delay and piecewise constant argument of generalized type. We have explored the stability and synchronization for the proposed neural network model via introduction of an impulsive control. We have presented the conditions for the stability of the network under some mild conditions, in contrast to the existing neural networks and the related dynamics, which needs additional conditions on synaptic weights for convergence. We have precisely investigated the convergence dynamics of the related dynamical system and have derived sufficient conditions for the global convergence. From application aspect, the proposed neural network model can be used to solve linear and convex quadratic programming problems. Thus, it possesses a remarkably extensive range of applications compared to the existing optimization neural networks and consequently, can be used to solve a wide range of optimization problems. Moreover, the convergence rate of our model is higher because the solution is global Mittag Leffler stable. 


\section{Acknowledgement}

We are thankful to the reviewers for their constructive comments, which helped in improving the manuscript.

\section{References}

[1] Nocedal J, Wright S. Numerical Optimization. Springer Science \& Business Media; 2006.

[2] Rao SS. Engineering Optimization: Theory and Practice. John Wiley \& Sons; 2009.

[3] Bazaraa MS, Sherali HD, Shetty CM. Nonlinear programming: Theory and Algorithms. John Wiley \& Sons; 2013.

[4] Effati S, Nazemi AR. Neural network models and its application for solving linear and quadratic programming problems. Applied Mathematics and Computation 2006; 172(1): 305-331.

[5] Tank DW, Hopfield JJ. Simple neural optimization networks: An A/D converter, signal decision circuit, and a linear programming circuit. IEEE Transactions on Circuits and Systems 1986; 33(5): 533-541.

[6] Kennedy MP, Chua LO. Neural networks for nonlinear programming. IEEE Transactions on Circuits and Systems 1988; 35(5): 554-562.

[7] $\mathrm{Hu} \mathrm{X}$. Applications of the general projection neural network in solving extended linearquadratic programming problems with linear constraints. Neurocomputing 2009; 72(4): 11311137.

[8] Xia Y, Leung H, Wang J. A projection neural network and its application to constrained optimization problems. IEEE Transactions on Circuits and Systems I: Fundamental Theory and Applications 2002; 49(4): 447-458.

[9] Zhang S, Constantinides AG. Lagrange programming neural networks. IEEE Transactions on Circuits and Systems II: Analog and Digital Signal Processing 1992; 39(7): 441-452.

[10] Effati S, Ranjbar M. A novel recurrent nonlinear neural network for solving quadratic programming problems. Applied Mathematical Modelling 2011; 35(4): 1688-1695.

[11] Yang Y, Cao J, Xu X, Hu M, Gao Y. A new neural network for solving quadratic programming problems with equality and inequality constraints. Mathematics and Computers in Simulation 2014; 101: 103-112.

[12] Xia YS, Wang J. On the stability of globally projected dynamical systems. Journal of Optimization Theory and Applications. 2000; 106(1): 129-150.

[13] $\mathrm{Hu}$ X, Wang J. Solving pseudomonotone variational inequalities and pseudoconvex optimization problems using the projection neural network. IEEE Transactions on Neural Networks $2006 ; 17(6)$ : 1487-1499. 
[14] Xia Y, Wang J. A recurrent neural network for solving linear projection equations. Neural Networks 2000; 13(3): 337-350.

[15] $\mathrm{Hu} \mathrm{X}$, Wang J. Design of general projection neural networks for solving monotone linear variational inequalities and linear and quadratic optimization problems. IEEE Transactions on Systems, Man, and Cybernetics, Part B: Cybernetics 2007; 37(5): 1414-1421.

[16] Xia Y, Wang J. A general projection neural network for solving monotone variational inequalities and related optimization problems. IEEE Transactions on Neural Networks 2004; 15(2): $318-328$.

[17] Liu Q, Yang Y. Global exponential system of projection neural networks for system of generalized variational inequalities and related nonlinear minimax problems. Neurocomputing 2010; 73(10): 2069-2076.

[18] Xue X, Bian W. A project neural network for solving degenerate convex quadratic program. Neurocomputing 2007; 70(13): 2449-2459.

[19] Xue X, Bian W. A project neural network for solving degenerate quadratic minimax problem with linear constraints. Neurocomputing 2009; 72(7): 1826-1838.

[20] Liu Q, Cao J. Global exponential stability of discrete-time recurrent neural network for solving quadratic programming problems subject to linear constraints. Neurocomputing 2011; 74(17): 3494-3501.

[21] Li F. Delayed Lagrangian neural networks for solving convex programming problems. Neurocomputing 2010; 73(10): 2266-2273.

[22] Srivastava HM, Lin SD, Chao YT, Wang PY. Explicit solutions of a certain class of differential equations by means of fractional calculus. Russian Journal of Mathematical Physics 2007; 14(3): 357-365.

[23] Lin SD, Tu ST, Srivastava HM, Wang PY. Certain operators of fractional calculus and their applications to differential equations. Computers \& Mathematics with Applications 2002; 44(12): $1557-1565$.

[24] Zhang S, Yu Y, Hu W. Robust stability analysis of fractional-order Hopfield neural networks with parameter uncertainties. Mathematical Problems in Engineering 2014; Article ID 302702, 14 pages, doi: $10.1155 / 2014 / 302702$.

[25] Wu ZB, Zou YZ. Global fractional-order projective dynamical systems. Communications in Nonlinear Science and Numerical Simulation 2014; 19(8): 2811-2819.

[26] Friesz TL, Bernstein D, Mehta NJ, Tobin RL, Ganjalizadeh S. Day-to-day dynamic network disequilibria and idealized traveler information systems. Operations Research 1994; 42(6): 11201136 .

[27] Wu H, Shi R, Qin L, Tao F, He L. A nonlinear projection neural network for solving interval quadratic programming problems and its stability analysis. Mathematical Problems in Engineering 2010; Article ID 403749, 13 pages, doi:10.1155/2010/403749.. 
[28] Abbas S. Existence and attractivity of k-pseudo almost automorphic sequence solution of a model of bidirectional neural networks. Acta Applicandae Mathematicae 2012; 119(1): 57-74.

[29] Abbas S, Yonghui X. Existence and attractivity of k-almost automorphic sequence solution of a model of cellular neural networks with delay. Acta Mathematica Scientia 2013; 33(1): 290-302.

[30] Abbas S. Pseudo almost periodic sequence solutions of discrete time cellular neural networks. Nonlinear Analysis, Modeling and Control 2009; 14(3): 283-301.

[31] Kinderlehrer D, Stampacchia G. An introduction to variational inequalities and their applications. SIAM; 1980.

[32] Miller RK, Michel RK. Ordinary Differential Equations. Academic Press; 1982.

[33] Kilbas AA, Srivastava HM, Trujillo JJ. Theory and applications of fractional differential equations. Elsevier Science Limited; 2006.

[34] Chen J, Zeng Z, Jiang P. Global Mittag-Leffler stability and synchronization of memristorbased fractional-order neural networks. Neural Networks 2014; 51: 1-8.

[35] Stamova I. Global Mittag-Leffler stability and synchronization of impulsive fractional-order neural networks with time-varying delays. Nonlinear Dynamics 2014; 77(4): 1251-1260. 